\title{
El suelo y su multifuncionalidad: ¿qué ocurre ahí abajo?
}

Montaño Arias, Noé Manuel; Navarro Rangel, María del Carmen; Patricio López, Itzel Carolina; Chimal Sánchez, Eduardo; Miguel de la Cruz, Jessica

El suelo y su multifuncionalidad: ¿qué ocurre ahí abajo?

CIENCIA ergo-sum, vol. 25, núm. 3, noviembre 2018-febrero 2019 | e31

Universidad Autónoma del Estado de México, México

Esta obra está bajo una Licencia Creative Commons Atribución-NoComercial-SinDerivar 4.0 Internacional.

Montaño Arias, N. M.; Navarro Rangel, M. del C.; Patricio López, I. C.; Chimal Sánchez, E. y Miguel de la Cruz, J. (2018). El suelo y su multifuncionalidad: ¿qué ocurre ahí abajo? CIENCIA ergo-sum, 25(3).

https://doi.org/10.30878/ces.v25n3a9 


\title{
El suelo y su multifuncionalidad: ¿qué ocurre ahí abajo?
}

The soil and its multifunctionality: What happens down there?

\author{
Noé Manuel Montaño Arias \\ Universidad Autónoma Metropolitana-Unidad Iztapalapa, \\ México \\ nmma@xanum.uam.mx \\ Maria del Carmen Navarro Rangel \\ Universidad Autónoma Metropolitana-Unidad Iztapalapa, \\ México \\ krmn.bio@gmail.com \\ Itzel Carolina Patricio López \\ Universidad Autónoma Metropolitana-Unidad Iztapalapa, \\ México \\ star79_@hotmail.com \\ Eduardo Chimal Sánchez \\ Universidad Autónoma Metropolitana-Unidad Iztapalapa, \\ México \\ chimalse31@gmail.com \\ Jessica Miguel de la Cruz \\ Universidad Autónoma Metropolitana-Unidad Iztapalapa, \\ México \\ miguelys44@gmail.com
}

Recepción: 10 de enero de 2017

Aprobación: 14 de julio de 2017

\section{Resumen:}

Se expone la definición del suelo y su relación con sus procesos de formación. Asimismo, se destaca y ejemplifica la importancia ambiental y agrícola del suelo como un sistema multifuncional esencial para la recirculación de los nutrimentos, hábitat de microbiodiversidad, almacén de carbono y como proveedor de alimentos y otros servicios ambientales cruciales para el mantenimiento de la biodiversidad del planeta. Finalmente, se discute sobre las actuales estrategias y perspectivas para su estudio en México, dado que se trata de un recurso natural no renovable y clave para nuestra seguridad ambiental y alimentaria.

Palabras clave: agronomía, biodiversidad del suelo, biogeociencias, ecología del suelo, ciencias del suelo.

\section{Abstract:}

We detail the soil definition in relation to the soil forming processes. We also emphasized and exemplified environmental and agricultural importance of the soil as an essential system with multifunctionality for cycling of nutrients, micro-biodiversity habitat, carbon stock, and as a supplier of food and other crucial environmental services for maintenance of the planet's biodiversity. Finally, the current strategies and prospects to study the soil in Mexico are discussed, since it is a natural nonrenewable resource and key to our environmental and food safety.

KEYWORDS: agronomy, soil biodiversity, biogeosciences, soil ecology, soil sciences.

\section{INTRODUCCIÓN}

El suelo no es algo que a la mayoría de las personas les resulte atractivo; sin embargo, aunque muchas veces no lo apreciamos, se trata de un recurso esencial para la sobrevivencia de todos los organismos en la Tierra, desde los microorganismos hasta el humano. El suelo es el soporte y el gran proveedor de alimento, ya que 
en él se almacenan los nutrimentos y el agua que sustentan a las plantas, incluidas las que cultivamos, por lo que es indispensable para el mantenimiento de la biodiversidad en todo el mundo. Con motivo de que la Organización de las Naciones Unidas para la Alimentación y la Agricultura (FAO por sus siglas en inglés) nombró el pasado 2015 como el Año Internacional del Suelo, el propósito de este artículo es explicar qué es el suelo, cómo se forma y qué servicios ambientales proporciona con la finalidad de concientizar sobre su estudio, multifuncionalidad en los agroecosistemas y ecosistemas y sobre su importancia ambiental como recurso natural enfatizando las perspectivas para su conservación y prioridades de investigación en México.

\section{QuÉ ES Y CÓMO SE FORMA EL SUELO}

El suelo es una delicada alfombra que recubre la corteza del planeta y que varía en grosor desde unos pocos centímetros hasta varios metros. Idóneamente, contiene minerales (45\%) derivados de la roca (arenas, limos y arcillas), aire (25\%), agua (25\%) y materia orgánica (5\%) que resulta de la descomposición de restos vegetales, animales y microbianos. La formación del suelo es producto de la continua interacción de cinco factores principales: $a$ ) el clima, $b$ ) los organismos vivos, $c$ ) el relieve, $d$ ) el tiempo y $e$ ) la roca madre o material parental. $\mathrm{Su}$ formación comienza cuando la roca se fragmenta y la materia orgánica se transforma por la acción de factores: a) físicos como la lluvia, el viento o la radiación solar, $b$ ) químicos que implican la reacción de sus constituyentes con el agua, el oxígeno y otros elementos químicos y $c$ ) biológicos producto de la actividad de los seres vivos (Porta et al., 2014). Al primero se le conoce como intemperismo de la roca y a los segundos como descomposición, ambos dan como resultado a largo plazo la liberación de minerales y elementos químicos (nutrimentos), los cuales pueden ser transportados por el viento o el agua de un lugar a otro en el relieve, o bien acumularse y conformar después de cientos o miles de años diferentes capas horizontales, denominadas horizontes del suelo, en donde el número y tipo de horizontes que se presentan y su espesor dependen del factor tiempo y de cinco procesos básicos para su desarrollo: $a$ ) las adiciones, $b$ ) pérdidas, $c$ ) transformaciones, $d$ ) translocaciones y e) turbaciones de los componentes minerales y orgánicos del suelo. Los horizontes se nombran con las letras $\mathrm{O}, \mathrm{A}, \mathrm{B}, \mathrm{C}$ y $\mathrm{R}$, cuyo orden indica desde el horizonte más superficial donde se acumula la materia orgánica (e.g. hojarasca, ramas, corteza, animales muertos, etc.) hasta el más profundo donde se encuentra la roca (Gardi et al., 2014; Porta et al., 2014; Brady y Weil, 2017 (figura 1).

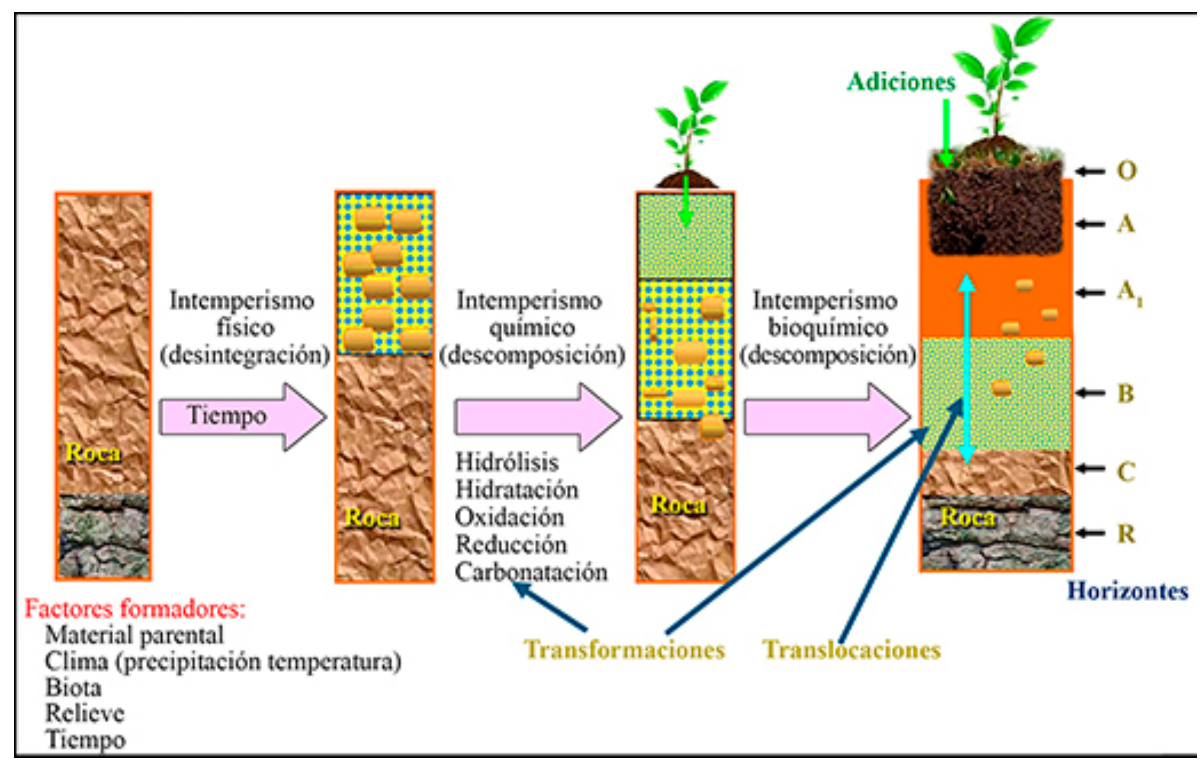

FIGURA 1

Esquema que representa los procesos y factores de la formación de un suelo Fuente: elaborado por N. M. Montaño y E. Chimal. 
Los horizontes están interconectados y s e ven continuamente a fectados por a diciones, transformaciones, translocaciones y pérdidas de materiales orgánicos e inorgánicos. Al conjunto de estos horizontes se le conoce como perfil edafológico, el cual se puede visualizar completo cuando se cava un pozo. El perfil permite también reconocer horizontes intermedios y de transición, producto del movimiento de materiales minerales y orgánicos (translocaciones), desde los horizontes profundos y superficiales hacia el centro del perfil. Así, cuando en un perfil se observa el horizonte B significa que el suelo está desarrollado, ya que éste posee componentes tanto orgánicos como inorgánicos muy finos; sin embargo, su formación es muy lenta, pues un sólo centímetro de suelo puede tardar hasta 1000 años en formarse, por lo que el suelo se considera un recurso natural no renovable en nuestra escala del tiempo (Porta et al., 2014; Brady y Weil, 2017). De este modo, en el suelo transitan diversos materiales orgánicos e inorgánicos, el agua, los nutrimentos, la energía y una enorme diversidad de organismos, los cuales conforman complejas interacciones que hacen del suelo un sistema con múltiples funciones en los ecosistemas.

\section{EL SUELO COMO FUENTE DE NUTRIMENTOS}

Los distintos constituyentes del suelo son fuente importante de nutrimentos para los organismos y de ellos depende la fertilidad edáfica de un ecosistema o campo agrícola. Sus componentes minerales y orgánicos proporcionan fósforo $(\mathrm{P})$, potasio $(\mathrm{K})$, calcio $(\mathrm{Ca})$, magnesio $(\mathrm{Mg})$, sodio $(\mathrm{Na})$, azufre $(\mathrm{S})$, nitrógeno $(\mathrm{N})$ y carbono $(\mathrm{C})$, entre otros, los cuales son necesarios para el crecimiento de las plantas y otros organismos como la fauna y microorganismos del suelo (Paul, 2007). La fauna tiene la peculiaridad de fragmentar 1 a materia orgánica y los microorganismos de metabolizar los compuestos orgánicos (carbono orgánico) transformándolos a formas químicas inorgánicas que pueden ser aprovechables por las plantas o incluso por ellos mismos (Orgiazzi et al., 2016); no obstante, algunos nutrimentos pueden reaccionar con otros compuestos químicos del suelo y quedar en formas inorgánicas no disponibles, como es el caso del $\mathrm{P}$ cuando reacciona con el carbonato de calcio o con el aluminio (Paul, 2007; Schlesinger, 2000; Brady y Weil, 2017). Sin embargo, su función en las transformaciones de la materia orgánica y en la liberación de nutrimentos no sería posible sin la participación del agua y del aire que están en los poros y canales del suelo, y que son indispensables para la actividad de la microbiota del suelo (Bardgett et al., 2005; Porta et al., 2014).

\section{3. ¿HAY ALGUien aHí ABaJo?}

El suelo está habitado por una infinidad de organismos misteriosos y fascinantes, los cuales se clasifican principalmente por su tamaño. Unos se aprecian a simple vista (macrofauna) como los milpiés, ciempiés, lombrices, arañas, cochinillas, hormigas, así como la mesofauna, compuesta por ácaros, colémbolos, nematodos y otros que no son visibles ante nuestros ojos como los protozoarios, los rotíferos (microfauna), hongos, archeas, actinomicetos y bacterias (microorganismos), ya que sólo pueden ser observados mediante un microscopio (Orgiazzi et al., 2016) (figura 2). Por ejemplo, en un metro cuadrado de suelo se pueden e ncontrar hasta 1000 especies de fauna edáfica y en un "puño" de suelo más microorganismos que seres humanos en el planeta. 


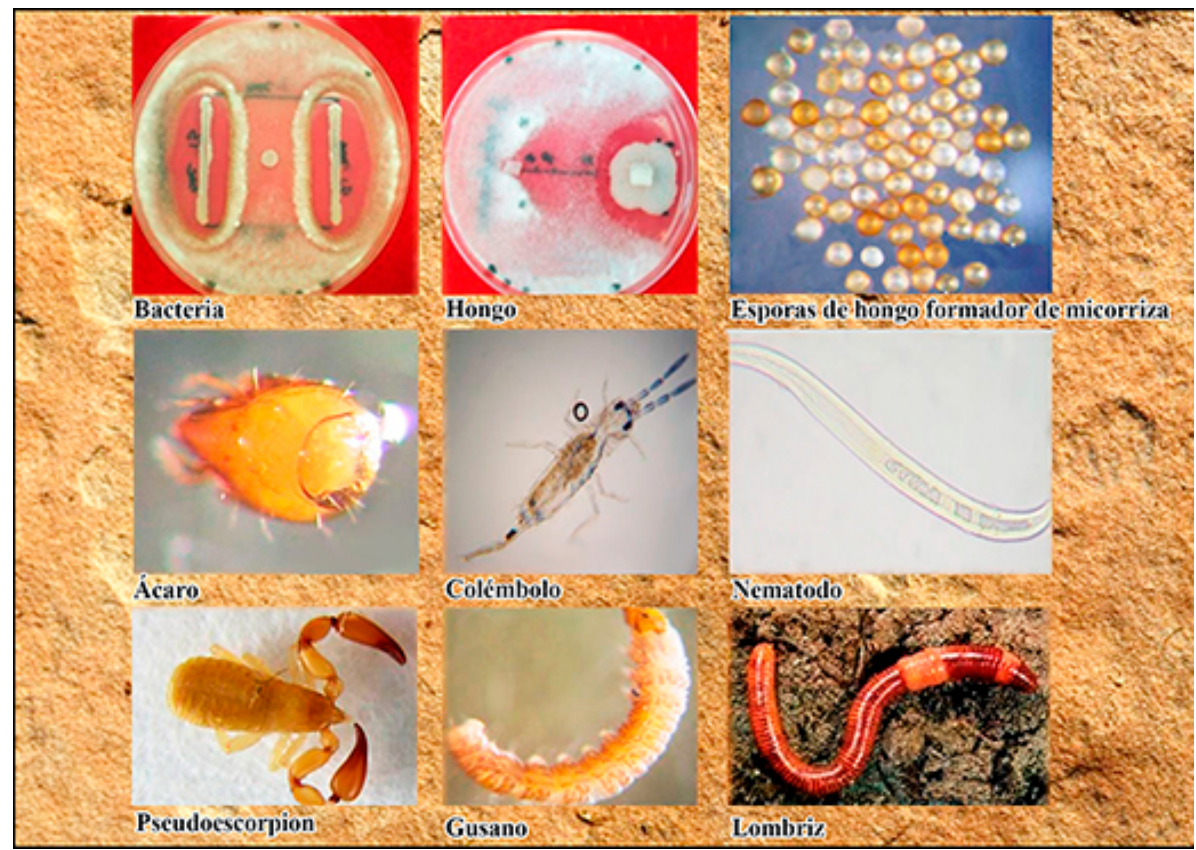

FIGURA 2

El suelo como un reservorio de fauna y microorganismos Fuente: fotos de J. Miguel.

Por ejemplo, por cada metro cuadrado de suelo se han estimado 350 millones de especies de bacterias, 63 especies de protozoarios, 4.5 millones de amebas, 50 ciempiés, 500 lombrices, 1.9 millones de nematodos, 745 termitas y hasta 2150 hormigas (Fragoso y Rojas, 2010). Con esto, los suelos albergan una cuarta parte de la biodiversidad del mundo y todas las especies que lo habitan establecen entre ellas y con las plantas complejas interacciones biológicas que van desde el parasitismo hasta el mutualismo y que en conjunto desempeñan funciones que permiten la transformación y recirculación de los nutrimentos en los ecosistemas (Paul, 2007). Estas interacciones y su función son tan importantes que se sabe, por ejemplo, que la eliminación selectiva de ácaros podría afectar de modo negativo la mineralización del $\mathrm{N}$ debido al incremento indirecto de las poblaciones de hongos (Orgiazzi et al., 2016). A pesar de esta información, aún se conoce muy poco de la biodiversidad del suelo, ya que menos de $1 \%$ de los microrganismos son cultivables en el laboratorio y sólo cerca de $20 \%$ de las especies de invertebrados han sido descritas; sin embargo, en la actualidad las técnicas moleculares están contribuyendo sustancialmente a conocer más organismos, a vincularlos con su función en el suelo y a detectar que incluso son tan vulnerables a extinguirse como los macroorganismos, lo cual tendría consecuencias negativas en el funcionamiento del suelo (Veresoglou et al., 2015).

\section{AHí ABAJo TRABAJAN EN EQUiPo}

La actividad conjunta de la fauna y microorganismos hace posible mantener los suelos saludables. En este sentido, por ejemplo, los caracoles y babosas, así como algunos hongos, secretan sustancias que al actuar como agentes cementantes fomentan la agregación de las partículas minerales y orgánicas del suelo y evitan su erosión; por su parte, las lombrices al crear microtúneles favorecen la aireación, infiltración del agua, penetración radicular y el transporte de nutrimentos (Fragoso y Rojas, 2010; Orgiazzi et al., 2016). Otro caso son los hongos que producen compuestos químicos que disuelven minerales de las rocas a formas químicas que puedan ser asimilables por otros organismos (Schlesinger, 2000; Paul, 2007). La micorriza es un claro ejemplo del trabajo en equipo; en este tipo de simbiosis mutualista, algunos hongos del suelo se asocian con las raíces de $80 \%$ de las familias de plantas, pues actúan como extensiones de éstas y ambos obtienen beneficios 
nutrimentales, además de permitir la retención y recirculación de los nutrimentos en el suelo (Bardgett et al., 2005). Esto ilustra cómo la microbiota del suelo realiza múltiples funciones que mantienen al ecosistema, a los cultivos de los campos agrícolas y a los suelos saludables permitiendo la coexistencia de la diversidad biológica y a su vez la producción de alimento.

\section{DiVERSIDAD DE SUELOS Y PRODUCCIÓN DE ALIMENTO}

La disponibilidad de alimentos está ligada a la diversidad de los suelos, pues de las características morfológicas, funcionales y del estado de salud del suelo depende la producción de frutos y granos, tanto en cantidad como en calidad nutrimental. Los suelos aportan 95\% de los alimentos a todos los organismos, incluidos los humanos a través de proveer con nutrimentos y agua a las plantas silvestres y cultivadas (Gardi et al., 2014; Porta et al., 2014). No obstante, el crecimiento mundial de la población humana ha fomentado día con día una mayor demanda de alimentos que son cultivados con tecnología agrícola -no amigable con el ambiente- y que deteriora las propiedades físicas, químicas y biológicas del suelo, la cual pone en riesgo su fertilidad y promueve como consecuencia su degradación (Gardi et al., 2014). En México existen 26 de los 32 grupos de suelo reconocidos por el Sistema Internacional Base Referencial Mundial del Recurso Suelo (IUSS, 2014), entre éstos dominan los Leptosoles (28.3\% del territorio), Regosoles (13.7\%), Phaeozems (11.7\%), Calcisoles (10.4\%), Luvisoles (9\%) y Vertisoles (8.6\%), los cuales ocupan $82 \%$ de la superficie nacional (Krasilnikov et al., 2011); sin embargo, es necesario hacer énfasis en su conservación dada su preponderante función en la producción de alimentos, ya que $44 \%$ de la superficie de México presenta actividad agrícola (Reyes-Jaramillo y Montaño, 2015) e indica que desde 2010, que es cuando se alcanza este porcentaje, existe una mayor presión sobre el recurso suelo. En consecuencia, es necesario atender la relación que existe entre la diversidad de los suelos y la producción de alimentos, ya que $38 \%$ de las causas de la degradación de los suelos en el país involucra actividades agropecuarias, mientras que otras están asociadas a la pérdida de vegetación por deforestación, sobreexplotación, urbanización o industrialización (Krasilnikov et al., 2011). Estudiar y comprender el funcionamiento del suelo para desarrollar sistemas de producción sustentables acordes con la conservación del suelo y la biodiversidad que alberga es una prioridad nacional para garantizar nuestra seguridad alimentaria (figura 3).

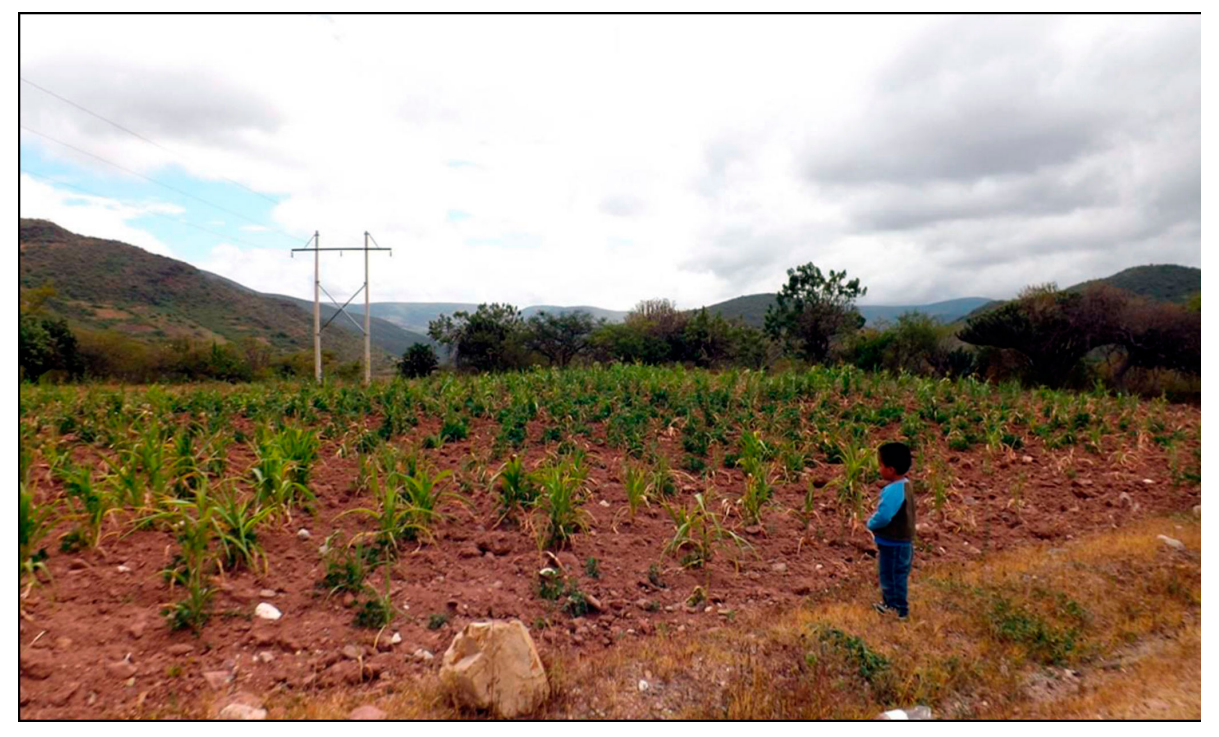

FIGURA 3

El suelo: el gran proveedor de alimento y la esperanza del futuro Fuente: foto de E. Chimal. 


\section{El suelo como almacén de CARbono}

El carbono (C) es la principal fuente de energía para las comunidades microbianas (archeas, bacterias, hongos, actinomicetos y otros, ya que durante la descomposición de la materia orgánica utilizan el C y otros nutrimentos para su propio desarrollo; sin embargo, el C no sólo está en el suelo, sino también en las plantas, animales, atmósfera y océanos (Schlesinger, 2000. Cuando la cobertura vegetal de un suelo es transformada a un área de cultivo, deforestada o quemada también se alteran los componentes del suelo, entre ellos el $\mathrm{C}$ que es liberado a la atmósfera, en donde se acumula en forma de dióxido de carbono $\left(\mathrm{CO}_{2}\right.$, un gas que intensifica el efecto invernadero y contribuye al calentamiento global del planeta (Johnston $e t$ al., 2004; Powlson et al., 2011; Crowther et al., 2016. En contraste, los suelos con mayor cobertura vegetal tienen más posibilidad de capturar $\mathrm{CO}_{2}$ debido a que las plantas lo incorporan a su biomasa por medio de la fotosíntesis y después al suelo a través del aporte de hojas, troncos, frutos, ramas y raíces y de su subsecuente descomposición (Johnston et al., 2004; Orgiazzi et al., 2016).

El suelo en los ecosistemas terrestres es el almacén más importante de $\mathrm{C}$ con hasta 2500 gigatoneladas y contiene hasta tres veces más $C$ que la vegetación o la atmósfera (Schlesinger, 2000). Así, en términos de mitigar el $\mathrm{CO}_{2}$ y su efecto en el cambio climático global, una acción prioritaria y necesaria es conservar el C en la vegetación, pero sobre todo en el suelo (Johnston et al., 2004; Milne et al., 2015; Crowther et al., 2016; Lefvre et al., 2017), ya que la interacción de este elemento con las partículas minerales y su almacenamiento en formas orgánicas complejas y de difícil descomposición (e.g. ligninas y ácidos húmicos) lo convierten en un reservorio estable a largo plazo debido a su lento reciclaje que ocurre de decenas a centenas de años (Schlesinger, 2000; Powlson et al., 2011; Crowther et al., 2016; Lefvre et al., 2017). Aparte de esto, la estructura del suelo, definida por el arreglo de sus partículas en agregados, es otro mecanismo que protege físicamente al $\mathrm{C}$ del ataque microbiano y lo conserva en el suelo por largos periodos (Lefvre et al., 2017). Esta misma situación ocurre con el C asociado a la elevada presencia de Ca y Mg que conforma unalmacén importante de $\mathrm{C}$ inorgánico en forma de carbonatos en el suelo de algunos ecosistemas como los áridos y semiáridos (Porta et al., 2014; Brady y Weil, 2017). En México, MurrayTortarolo et al. (2016) estimaron los almacenes de C mediante datos de satélite, mediciones en campo, modelos espaciales y torres de monitoreo. Estos autores reportaron un almacén total de C de $34506 \pm 7$ 483 teragramos, con $20347 \pm 4622$ almacenados en la vegetación y $14159 \pm 3861$ en el suelo, lo que indica que $70 \%$ del C está en el suelo. Asimismo, también se estima que los suelos de las regiones áridas y semiáridas del país almacenan hasta $37 \mathrm{Mg} \mathrm{ha}^{-1}$ de $\mathrm{C}$ orgánico y $17.9 \mathrm{Mg} \mathrm{ha}^{-1}$ de $\mathrm{C}$ inorgánico que representa $46 \%$ del $C$ total (Montaño et al., 2016), lo que sugiere que estas regiones, que cubren el $60 \%$ del territorio mexicano, son un importante almacén, en particular de $\mathrm{C}$ inorgánico. De este modo, ambos estudios indican que los suelos de México ofrecen un servicio ambiental crucial que es el secuestro de $\mathrm{C}$, por lo que contribuyen a la mitigación del $\mathrm{CO}_{2}$ de la atmósfera y del cambio climático.

\section{OTROS SERVICIOS AMBIENTALES DEL SUELO}

El suelo brinda otros servicios ambientales (Milne et al., 2015) (figura 4) como la generación de oxígeno a partir de dar soporte a los productores primarios, la regulación del clima y de los flujos hidrológicos esenciales en la recarga de acuíferos y el suministro de los nutrimentos que sustentan a las plantas nativas

o cultivadas, entre ellas una gran variedad de gramíneas y leguminosas que proveen de forrajes, fibras, biocombustibles, granos y frutos a los organismos. Además, suministra materiales para la construcción de vivienda e infraestructura (e.g. yeso, grava, arena, arcilla, cal), y también es un reservorio importante de herencia cultural (cerámicas de culturas milenarias) y biológica (registro fósil). Además, como ya se mostró, el suelo es el hábitat de miles de millones de microorganismos que constituyen un recurso genético esencial para 
el control biológico de plagas o para la producción de medicamentos como la penicilina o la estreptomicina, ambos relacionados con la cura o control de infecciones en humanos y que se obtienen a partir de la actividad metabólica del hongo Penicillium notatum y el actinomiceto Streptomycetes sp., respectivamente (Paul, 2007). El suelo es también, bajo ciertas circunstancias, un almacén y depurador de elementos contaminantes que son producto de actividades antrópicas y que por lo general son adicionados al suelo. Así, la presencia de metales pesados, hidrocarburos y plaguicidas, todos ellos potencialmente tóxicos, es uno de los problemas ambientales más importantes en el mundo. Varias especies de plantas, e incluso grupos microbianos que habitan en estos suelos, son cruciales en la biorremediación edáfica, ya que promueven en conjunto con la composición y características del suelo la descontaminación o estabilización de los contaminantes, en donde la cantidad del contaminante no disminuye, pero el elemento queda secuestrado o adsorbido en la matriz del suelo, por lo que su biodisponibilidad y movimiento sí se minimizan. Con esto, se considera que el suelo retiene, filtra y desactiva compuestos tóxicos que pueden llegar a los mantos freáticos y a las cadenas alimenticias y redes tróficas, puesto que es un recurso esencial para reducir los riesgos ambientales y de salud (Alarcón y Ferrera-Cerrato, 2013).

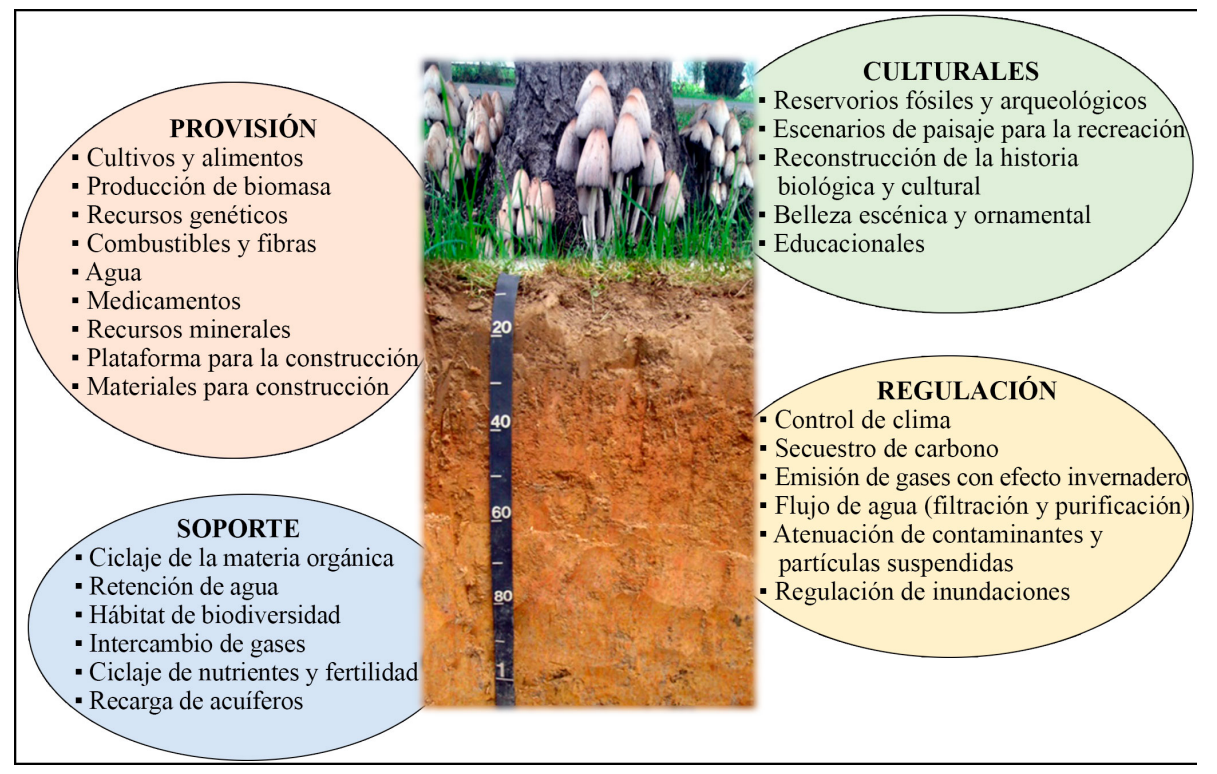

FIGURA 4

Servicios ambientales proporcionados por el suelo

Fuente: foto de N. M. Montaño y C. Patricio.

\section{Perspectivas sobre el recurso suelo en México}

Lo expuesto en este trabajo proyecta la necesidad actual de continuar estudiando el suelo. Si bien desde el siglo pasado su estudio aportó importantes avances para conocerlo y utilizarlo, hoy en día se ha convertido en un elemento de los ecosistemas clave de documentar para comprender casi cualquier otro sistema biológico vinculado directa o indirectamente al suelo. De este modo, es prioritario que licenciaturas y posgrados en agronomía, biología, ecología, ciencias ambientales, ciencias forestales y geociencias, mantengan y refuercen en sus planes de estudio las unidades de aprendizaje que permitan la formación de especialistas con un pleno conocimiento del suelo. Es también relevante promover la construcción de laboratorios que incluyan herramientas de biología molecular, bioquímica, análisis isotópico y microscopía electrónica que faciliten a los estudiantes e investigadores abordar la estructura y función del suelo a una escala más fina. Esto se 
convierte en una necesidad que queda en evidencia con el surgimiento de nuevas áreas transdisciplinares del conocimiento como las biogeociencias (Martin y Johnson, 2012), cuyo éxito en la solución de problemas ambientales requiere de considerar a los procesos biogeoquímicos que ocurren en el suelo desde el nivel genético hasta el ecosistémico a través de su interacción con otros sistemas como la atmósfera, así como con la creciente demanda de alimento y de servicios ambientales que dependen del suelo. Entonces, ahora más que nunca se requiere de una educación ambiental básica y superior que reconozca el suelo como el ambiente en común que interconecta a todos los elementos que integran a los ecosistemas y agroecosistemas, y a partir de ello implementar una política pública que visualice la multifuncionalidad del suelo (Reyes-Sánchez, 2015), promueva una mayor inversión en la investigación científica para su estudio y permita atender de manera efectiva la conservación y manejo sustentable de este recurso natural.

Aunque a gran escala se conoce relativamente bien la distribución de los suelos en México y sus múltiples interacciones ambientales (Krasilnikov et al., 2011), un reto importante en el corto y mediano plazo es fortalecer el inventario nacional de los suelos de México en términos de los servicios ambientales que éstos ofrecen y de priorizar su manejo sustentable con el objetivo de garantizar el sustento de la biodiversidad y la seguridad alimentaria. Es probable que una acción prioritaria sea la inversión para generar más conocimiento sobre la biota del suelo y sus funciones para poder utilizarla como una biotecnología aplicable, tanto desde el punto de vista biotecnológico e industrial como ecológico y agrícola. Esta acción podría además combinarse con estrategias tradicionales del manejo del suelo, las cuales se deben rescatar desde el conocimiento de las culturas milenarias, ya que varias de éstas, como los sistemas agroforestales que aún manejan algunas etnias, han demostrado ser eficientes desde épocas prehispánicas en el uso y conservación del suelo, así como en preservar la biodiversidad en armonía con la producción agrícola (Moreno-Calles et al., 2016), por lo que el rescate y el apoyo al desarrollo de los sistemas tradicionales y del conocimiento local sobre el manejo del suelo deber ser también una prioridad nacional.

En la legislación mexicana el suelo se aprecia desde la dimensión territorial y productiva, en donde las normas oficiales en materia del suelo se enfocan al aprovechamiento sustentable y recuperación de este recurso, lo que contribuye a su conservación. En México existen instrumentos jurídicos transversales entre distintas secretarías y organismos gubernamentales (e.g. Semarnat, Sagarpa, Conafor) dirigidos a la conservación del suelo, pero su implementación práctica a través de programas locales o regionales se realiza muy pocas veces con muy bajos recursos económicos y la comunidad científica mexicana dedicada al estudio del suelo no es incluida en las decisiones legislativas y en su implementación (Cotler et al., 2007). Por estas razones, una política pública fincada en la educación a todos los niveles de la población y fundamentada en la investigación, difusión y capacitación debe convertirse en una prioridad gubernamental y de todos los ciudadanos, dado el papel del suelo en la seguridad alimentaria y como soporte de la biodiversidad del país.

\section{Conclusiones}

El suelo ofrece servicios ambientales como producto de su constitución física, química y de la actividad de una gran diversidad de organismos que lo habitan y que trabajan en equipo dando soporte a su multifuncionalidad, muchos incluso desconocidos por completo, pero con funciones posiblemente únicas para mantener la fertilidad. Comprender las funciones de la microbiota en los distintos suelos es un punto crítico, ya que de ella depende la recirculación de la materia orgánica, la liberación de nutrimentos, la filtración de elementos contaminantes, el mantenimiento de la macro-biodiversidad y la seguridad alimentaria de una nación. En consecuencia, es esencial estudiar el suelo y generar políticas públicas para su conservación y manejo sustentable, ya que se trata de un recurso natural no renovable en el corto tiempo, y que es crítico para el funcionamiento de los ecosistemas. Así, al pisar o alterar el suelo debemos recordar que ahí abajo ocurren múltiples funciones y que se trata de un micromundo complejo y bien estructurado que hace posible nuestra 
existencia y supervivencia y, por lo tanto, se debe considerar un bien de interés público que requiere de una atención efectiva por parte de las distintas instancias del gobierno y de la participación activa de la ciudadanía.

\section{ReFERENCiAS}

Alarcón, A. y Ferrera-Cerrato, R. (2013). Biorremediación de suelos y aguas contaminadas con compuestos orgánicos e inorgánicos. México: Editorial Trillas.

Bardgett, R., Usher, M. y Hopkins, P. (2005). Biological diversity and function in soils. USA: Cambridge University Press.

Brady, N. C. y Weil, R. R. (2017). The nature and properties of soils (15th ed.). Columbus: Pearson.

Cotler, H., Sotelo, E., Domínguez, J., Zorrilla, M., Cortina, S. y Quiñones, L. (2007). La conservación de suelos: un asunto de interés público. Gaceta Ecológica, 83, 5-71.

Crowther, T. W., Todd-Brown, K. E. O., Rowe, C. W., Wieder, W. R., Carey, J. C., Machmuller, M. B., Snoek, B. L., Fang, S., Zhou, G., Allison, S. D., Blair, J. M., Bridgham, S. D., Burton, A. J., Welker, J. M. y Bradford, M. A. (2016). Quantifying global soil carbon losses in response to warming. Nature, 104(540), 104-108.

Fragoso, C. y Rojas, P. (2010). La biodiversidad escondida: la vida microscósmica en el suelo, en Toledo V. M. (Coord.), La biodiversidad de México: inventarios, manejos, usos, informática, conservación e importancia cultural (pp. 90-134). México: Fondo de Cultura Económica.

Gardi, C., Angelini, M., Barceló, S., Comerma, J., Cruz-Gaistardo, J., Encina Rojas, A., Jones, A., Krasilnikov, P., Mendonça Santos Brefin, M. F., Montanarella, L., Muñiz Ugarte, O., Schad, P., Vara Rodríguez, M. I. y Vargas, R. (2014). Atlas de suelos de América Latina y el Caribe. Luxembourg, Comisión Europea, Unión Europea, L-2995.

IUSS (Grupo de Trabajo WRB). (2014). Base referencial mundial del recurso suelo. Informes sobre Recursos Mundiales de Suelos No. 103. Roma: FAO.

Johnston, C. A., Groffman, P., Breshears, D. D., Cardon, Z. G., Currie, W., Emanuel, W., Gaudinski, J., Jackson, R. B., Lajtha, K., Nadelhoffer, K., Nelson, Jr. D., Retallack, G., Post, W. M. y Wielopolski, L. (2004). Carbon cycling in soil. Frontiers in Ecology and Environment, 2(10), 522-528.

Krasilnikov, P., Jiménez-Nava, F. J., Reyna-Trujillo, T. y García-Calderón, N. E. (2011). Geografía de suelos de México. México: Las Prensas de Ciencias, UNAM.

Lefèvre, C., Rekik, F., Alcantara, V., Wiese, L. (2017). Soil organic carbon: the hidden potential. Rome: Food and Agriculture Organization (FAO) of the United Nations.

Martin, Y. E. y Johnson, E. A. (2012). Biogeociencies survey: studying interactions of the biosphere with the lithosphere, hydrosphere and atmosphere. Progress in Physical Geography, 36(6), 833-852.

Milne, E., Banwart, S. A., Noellemeyer, E., Abson, D. J, ... y Zheng, J. (2015). Soil carbon, multiples benefits. Environmental Development, 13, 33-38.

Moreno-Calles, A. I., Casas, A., Toledo, V. M. y Vallejo, M. (2016). Etnoagroforesteria en México. México: UNAM.

Montaño, N. M., Ayala, F., Bullock, S. H., Briones, O., García-Oliva, F., García-Sánchez, R., Maya, Y., Perroni, Y., Siebe, Ch., Tapia-Torres, Y., Troyo, E. y Yépez, E. (2016). Carbon stocks and fluxes in arid and semiarid ecosystems of Mexico: Synthesis and prospects. Terra Latinoamericana, 34(1), 39-59.

Murray-Tortarolo, G., Friedlingstein, P., Sitch, S., Jaramillo, V. J., Murgía-Flores, F., Anav, A., Liu, Y., Arneth, A., Arvanitis, A., Harper, A., Jain, A., Kato, E., Koven, C., Poulter, B., Stocker, B. D., Wiltchire, A., Zaehle, S. y Zeng, N. (2016). The carbon cycle in Mexico: past, present and future of C stocks and fluxes. Biogeosciences, 13, 223-238.

Orgiazzi, A., Bardgett, R. D., Barrios, E., Behan-Pelletier, V., Briones, M. J. I., Chotte, J-L., De Deyn, G. B., Eggleton, P., Fierer, N., Fraser, T., Hedlund, K., Jeffery, S., Johnson, N. C., Jones, A., Kandeler, E., Kaneko, N., Lavelle, P., Lemanceau, P., Miko, L., Montanarella, L., Moreira, F. M. S., Ramírez, K. S., Scheu, S., Singh, B. K., Six, J., van der Putten, W. H. y Wall, D. H. (2016). Global Soil Biodiversity Atlas. Luxembourg, European Commission, Publications Office of the European Union. 
Paul, E. A. (2007). Soil microbiology, ecology, and biochemistry. USA: Academic Press.

Porta, J., López-Acevedo, M. y Poch, R. M. (2014). Edafología: uso y protección de suelos. Madrid: Mundi-Prensa S. A. de C. V.

Powlson, D. S., Whitmore, A. P. y Goulding, K. W. T. (2011). Soil carbon sequestration to mitigate climate change: a critical reexamination to identify the true and the false. European Journal of Soil Science, 62, 42-55.

Reyes-Jaramillo, I. y Montaño, N. M. (2015). Diversidad de los suelos de México. La Jornada del Campo (suplemento), 91, 11-12.

Reyes-Sánchez, L. B. (2015). La educación como política pública de concientización para la preservación del suelo como recurso natural limitante para la existencia de la vida, en G. Cruz-Flores y A. M. López-López (Eds.),

Redescubriendo el suelo: su importancia ecológica y agrícola (pp. 5-17). México: UNAM-Conacyt.

Schlesinger, W. H. (2000). Biogeoquímica, un análisis global (tercera edición). Barcelona: Arielciencia.

Veresoglou, S. D., Halley, J. M. y Rillig, M. C. (2015). Extinction risk of soil biota. Nature Communications, 6, 8862-9862.

\section{BY-NC-ND}

